Available online on 15.05.2020 at http://ujpr.org
Universal Journal of Pharmaceutical Research
An International Peer Reviewed Journal
Open access to Pharmaceutical research is an open access article distributed under the terms of the Creative Commons Attribution-Non
Commercial Share Alike 4.0 License which permits unrestricted non commercial use,
provided the original work is properly cited
Volume 5, Issue 2, 2020

\title{
GREEN, RAPID, SIMPLE, AND AN EFFECTIVE ONE-POT MULTICOMPONENT STRATEGY FOR SYNTHESIS OF NOVEL DIHYDROPYRANO[2,3-C]PYRAZOL-6-AMINES IN AQUEOUS MEDIUM Sadeq Hamood Saleh Azzam ${ }^{1}$ (D) , Aisha Siddekha ${ }^{2,3}$ (D), MA Pasha ${ }^{*, 2}$ (1) \\ ${ }^{\prime}$ Department of Chemistry, Sana'a University, Sana' a, Yemen. \\ ${ }^{2}$ Department of Studies in Chemistry, Jnana Bharathi Campus, Bangalore University, Bangalore-5 60056, India. \\ ${ }^{3}$ Department of Chemistry, Government First Grade College, B.H. Road, Tumkur-572102, Karnataka, India.
}

\section{ABSTRACT}

Objectives: A series of new dihydropyrano [2,3-c]pyrazol-6-amines in this investigation study, are synthesized by the strategy of one-pot four component reaction in an aqueous medium.

Methods: After getting optimum conditions the reaction of different substituted aromatic aldehydes, ethyl acetoacetate, hydrazine hydrate, and phenylacetonitrile catalysed by Sodium hydrogen sulphate $\mathrm{NaHSO}_{4}(0.5 \mathrm{~m}$ mol) by refluxing in ethanol-water (1:1) $(5 \mathrm{ml})$ and heated at $80{ }^{\circ} \mathrm{C}$. The synthesized molecules product was filtered and washed with water to get nearly pure product in excellent yield.

Results: The pure products which were got in a short duration, with excellent yield $(80-87 \%)$. Those new compounds were identified by using spectroscopic techniques FT-IR, ${ }^{1} \mathrm{H}-\mathrm{NMR}$ and ${ }^{13} \mathrm{C}-\mathrm{NMR}$.

Conclusion: In conclusion, the usefulness of this one-pot multicomponents protocol include easily available starting materials, facile, simple and environment-friendly procedure, and easier separation of products by filtration.

Keywords: Araldehydes, ethyl acetoacetate, fluoro phenylacetonitrile, hydrazine hydrate, pyrano[2,3-c]pyrazol-6-amines, water.

Article Info: Received 5 March 2020; Revised 3 April; Accepted 9 May, Available online 15 May 2020

$\square$ Cite this article-

Saleh Azzam SH, Siddekha A, Pasha MA. Green, rapid, simple, and an effective one-pot multicomponent strategy for synthesis of novel dihydropyrano[2,3-c]pyrazol-6-amines in aqueous medium. Universal Journal of Pharmaceutical Research 2020; 5(2):16-22.

DOI: https://doi.org/10.22270/ujpr.v5i2.383

Address for Correspondence

M. A. Pasha, Department of Studies in Chemistry, Central College Campus, Bangalore University, Bangalore-560 001, India,

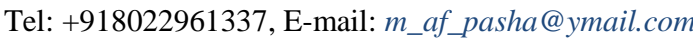

\section{INTRODUCTION}

In the last two decades, the rising environmental concern in chemistry has redressed spotlight on the multicomponent reactions strategy as new trends in organic synthesis chemistry ${ }^{1,2}$. Multicomponent reactions (MCRs) are very useful for the construction of huge numbers of heterocyclic compounds ${ }^{3}$, using this strategy biologically active substances and natural product compounds have been synthesized ${ }^{4}$.The nitrogen containing heterocycles synthesis is one of great interest field in organic synthesis because of they constitute an important class of natural and synthetic products, many of them which exhibit useful biological activity and find application in pharmaceutical preparations $^{5-11}$

The need to develop the concept of green chemistry to help safeguard human life was started after the first chemical revolution, which changed modern life with excellent entertainment and services, but also created the serious issue of environmental pollution. Thus, The essential principle of the green chemistry concept is to protect the environment, not only by cleaning it up, but by discovery and designing of new chemistry and pharmaceutical industries to concern the effect on human life when new chemicals are introduced into our society ${ }^{12-16}$. Compared to others green solvents water appears to be a better option because of its super abundant and non-inflammable nature non-toxic, noncorrosive. In addition, water can be governed because of its relatively high vapour pressure as compared to organic solvents, that making it a green and sustainable alternative ${ }^{17-20}$. Water offers several benefits such as control over exothermic reactions, salting in and salting out, as well as variation of $\mathrm{pH}$ range ${ }^{21}$. Recently, organic reactions in water without use of hurtful organic solvents have drawn much more attention, because water is cheap, safe and environment friendly solvent ${ }^{22,23}$. 
Dihydropyrano[2,3-c]pyrazole scaffold represents very motivating template in medicinal chemistry, and play an essential role as biological active molecules ${ }^{24}$. Many of the pyrano[2,3-c]pyrazoles are known for their antimicrobial ${ }^{25}$, insecticidal ${ }^{26}$, anti-inflammatory ${ }^{27}$, anticancer $^{28}$ and molluscicidal activities ${ }^{29}$. During the last few years, synthesis of dihydropyrano[2,3c]pyrazoles has received major interest $\mathrm{t}^{30,31}$.

Pyranopyrazoles are also used as pharmaceutical ingredients and biodegradable agrochemicals ${ }^{32}$. Derivatives of dihydropyrano[2,3-c]pyrazole possess useful pharmacological and biological properties ${ }^{33}$. Only a few reports are available on the synthesis of dihydropyrano[2,3-c]pyrazol-6-amines ${ }^{34,35}$. A FourComponent Domino Combinatorial Synthesis in presence of DIPEA is reported by Kanchithalaivan et $a l .{ }^{36}$. In continuation of current efforts to find and develop a new routes for the synthesis of biologically active heterocyclic molecules using inexpensive, readily available, and environmentally benign catalysts $^{37-43}$, thus herein, we wish to report a mild, efficient method for the synthesis of some novel and simple one-pot four-component synthesis of dihydropyrano [2, 3-c] pyrazol-6-amines using green chemistry principles. In the present study, a series of novel dihydropyrano [2,3-c]pyrazol-6-amines (5) are synthesized by a one-pot four-component reaction of hydrazine hydrate (4), araldehydes, ethyl acetoacetate (2), and 4-fluoro phenylacetonitrile (3) in water using sodium hydrogen sulphate as a catalyst in good yields as shown in the following Figure 1.<smiles>[R]c1ccccc1C1C(c2ccc(F)cc2)=C(N)Oc2[nH]nc(NCNN)c2C1c1ccccc1</smiles>

Figure 1: Synthesis of dihydropyrano $[2,3-c]$ pyrazol-6-amines.

\section{MATERIALS AND METHODS}

Chemicals of analytical grade were arranged locally. The chemicals that has been used were commercial (SD fine Company AR grade) and without further purification. The progress of the reaction was monitored on TLC (Eluent; 2:8 ethyl acetatepetroleum ether). Melting points were measured in open capillary tubes and are uncorrected using Raaga, melting point apparatus, Indian make. FT-IR spectra were recorded on SHIMADZU FT-IR-8400s spectrophotometer (Shimadzu Company). ${ }^{1} \mathrm{H}$ NMR and ${ }^{13} \mathrm{C}$ NMR spectra of the synthesised products were recorded on Bruker AMX $400 \mathrm{MHz}$ (Bruker Company) and $100 \mathrm{MHz}$ respectively in DMSO- $d_{6}$ as solvent and TMS as internal standard reference.

General procedure for preparation of dihydropyrano [2,3-c]pyrazol-6-amines :

The mixture of aromatic aldehyde $(1 \mathrm{~m} \mathrm{~mol})$, malononitrile $(1 \mathrm{~m} \mathrm{~mol})$, ethyl acetoacetate $(1 \mathrm{~m} \mathrm{~mol})$ hydrazine hydrate $(1 \mathrm{~m} \mathrm{~mol})$ and $\mathrm{NaHSO}_{4}(0.5 \mathrm{~m} \mathrm{~mol})$ were taken in ethanol-water $(1: 1)(5 \mathrm{ml})$ and heated at $80{ }^{\circ} \mathrm{C}$. The progress of the reaction was monitored using Silica gel-G TLC plates with a mixture of petroleum ether $\left(60-80^{\circ} \mathrm{C}\right)$ and ethyl acetate $(20: 80)$ as eluent. After the reaction has been completed, the mixture was cooled to room temperature and the precipitated product was filtered, washed with water to get nearly pure product.

\section{RESULTS AND DISCUSSION}

At the beginning, to identify the optimum reaction conditions, a representative reaction between 3methoxy, 4-hydroxy benzaldehyde (vanillin), ethylacetoacetate, 4-fluoro phenylacetonitrile and hydrazine hydrate (1 m mol each) was considered. To begin with, the reaction was carried out without any catalyst in ethanol, at room temperature, ultrasonic and microwave irradiation which didnot afford desired product. This test reaction was then probed using different catalysts in refluxing ethanol which gave different product yields. In present study, $\mathrm{NaHSO}_{4}$, the reaction afforded better yield when compared to other catalysts (Table 1).

Table 1: Selection of suitable catalyst and solvent for the synthesis of $(5 \mathrm{~d})^{\mathrm{a}}$.

\begin{tabular}{clccc}
\hline Entry & $\begin{array}{l}\text { Catalyst } \\
(\mathbf{1 0} \text { mol \%) }\end{array}$ & Solvent & $\begin{array}{c}\text { Time } \\
(\mathbf{m i n})\end{array}$ & $\begin{array}{c}\text { Yield }^{\text {a }} \\
(\mathbf{\%})\end{array}$ \\
\hline 1 & Imidazole & Ethanol & 120 & 45 \\
2 & $\mathrm{NaOH}$ & Ethanol & 140 & 50 \\
3 & $\mathrm{Ba}(\mathrm{OH})_{2}$ & Ethanol & 130 & 45 \\
4 & $\mathrm{NaHSO}_{4}$ & Ethanol & 120 & 55 \\
5 & $\mathrm{NaHSO}_{4}$ & Water & 120 & 50 \\
6 & $\mathrm{NaHSO}_{4}$ & $\begin{array}{c}\text { Water- } \\
\text { ethanol(1:1) }\end{array}$ & 65 & 87 \\
7 & $\mathrm{NaHSO}_{4}$ & Acetonitrile & 120 & 40 \\
\hline \multicolumn{5}{c}{${ }^{a}$ isolated yields } \\
\end{tabular}

After selection of catalyst for the reaction, investigation for an appropriate solvent was performed. The representative reaction was carried out in solvents such as ethanol, water, acetonitrile and water: ethanol (1: 1) mixture (Table 1). As seen from the data in Table 1, Water-ethanol (1:1) was found to be the ideal solvent for this reaction which afforded maximum yield of the product.

Table 2: Effect of the amount of $\mathrm{NaHSO}_{2}$ on the synthesis of $(5 \mathrm{e})^{\mathrm{a}}$.

\begin{tabular}{lcc}
\hline Entry & $\begin{array}{c}\mathbf{N a H S O}_{\mathbf{4}} \\
(\mathbf{m o l} \%)\end{array}$ & $\begin{array}{c}\text { Yield } \\
(\boldsymbol{\%})\end{array}$ \\
\hline 1 & 5 & 50 \\
2 & 7 & 65 \\
3 & 10 & 89 \\
4 & 12 & 89 \\
\hline
\end{tabular}

${ }^{\mathrm{a}}$ Reactions are performed on a $1 \mathrm{mmol}$ scale of the reactants. 
The explanation of the difference between those solvents is due to their relative polarity which indicates water (1.0) is the most polar solvent among them then ethanol (0.654) less than water and acetonitrile (0.460) the least polar among them, thus the mixture of water and ethanol (1: 1) made the polarity of water ethanol is proper for the reaction and gives the best result as well as easily to remove them from the product by washing. Acetonitrile is not proper solvent which gives low yield of the product due to its low relative polarity and difficult to remove from product it needs sophisticated means to remove from the product. The reaction template, with $\mathrm{NaHSO}_{4}$ as catalyst and Water-ethanol (1:1) as a solvent system, was applied to a different substituted araldehydes to prepare a library of compounds. The template works well for all the araldehydes to give corresponding products (Table 3 ).

Table 3: Synthesis of novel dihydropyrano [2,3-c]pyrazol-6-amines from various aldehydes, hydrazine hydrate, ethyl acetoacetate, and 4-chloro phenylacetonitrile and $\mathrm{NaHSO}_{4}$ in ethanol-water (1:1) solvent.

\begin{tabular}{|c|c|c|c|c|c|}
\hline Entry & Aldehyde (1) & Product & $\begin{array}{l}\text { Time } \\
(\mathrm{min})\end{array}$ & $\begin{array}{c}\text { Yield }^{\mathrm{a}} \\
(\%)\end{array}$ & $\begin{array}{c}\text { MP }\left({ }^{\circ} \mathrm{C}\right) \\
\text { found }\end{array}$ \\
\hline 1. & $3-\mathrm{NO}_{2} \mathrm{C}_{6} \mathrm{H}_{4} \mathrm{CHO}$ & $5 a$ & 120 & 85 & $185-187$ \\
\hline 2. & $4-\mathrm{HOC}_{6} \mathrm{H}_{4} \mathrm{CHO}$ & $5 b$ & 120 & 82 & $236-239$ \\
\hline 3. & $4-\mathrm{ClC}_{6} \mathrm{H}_{4} \mathrm{CHO}$ & $5 c$ & 120 & 80 & $199-200$ \\
\hline 4. & $3-\mathrm{MeO}, 4-\mathrm{HOC}_{6} \mathrm{H}_{3} \mathrm{CHO}$ & $5 d$ & 80 & 87 & $260-263$ \\
\hline 5. & $3-\mathrm{MeOC}_{6} \mathrm{H}_{4} \mathrm{CHO}$ & $5 e$ & 80 & 89 & $195-197$ \\
\hline 6. & $3,4,5-(\mathrm{MeO})_{3} \mathrm{C}_{6} \mathrm{H}_{2} \mathrm{CHO}$ & $5 f$ & 90 & 80 & $180-184$ \\
\hline 7. & $4-\mathrm{NO}_{2} \mathrm{C}_{6} \mathrm{H}_{4} \mathrm{CHO}$ & $5 g$ & 90 & 82 & $271-273$ \\
\hline 8. & $2,4-\mathrm{Cl}_{2} \mathrm{C}_{6} \mathrm{H}_{3} \mathrm{CHO}$ & $5 \mathrm{~h}$ & 120 & 80 & 205-207 \\
\hline 9. & 3,4-(MeO $)_{2} \mathrm{C}_{6} \mathrm{H}_{3} \mathrm{CHO}$ & $5 \mathrm{i}$ & 120 & 80 & $175-178$ \\
\hline 10. & $2-\mathrm{HOC}_{6} \mathrm{H}_{4} \mathrm{CHO}$ & $5 \mathrm{j}$ & 90 & 82 & $200-204$ \\
\hline 11. & $\mathrm{HCHO}$ & $5 \mathrm{k}$ & 240 & ND & - \\
\hline 12. & $\mathrm{CH}_{3} \mathrm{CHO}$ & 51 & 240 & ND & - \\
\hline 13. & $\mathrm{C}_{6} \mathrm{H}_{5} \mathrm{CH}=\mathrm{CHCHO}$ & $5 \mathrm{~m}$ & 240 & ND & - \\
\hline
\end{tabular}

The reaction template failed for the using of aliphatic aldehydes (Table 3, entries 11-12) and the $\alpha, \beta$ unsaturated aldehydes such as cinnamaldehyde (Table 3 , entry 13) as well did not give any product even after the reaction was carried out for longer duration. To select a best catalyst and proper solvent, we carried out the reaction between 3-methoxy, 4-hydroxy benzaldehyde (vanillin), ethylacetoacetate, 4-fluoro phenylacetonit-rile and hydrazine hydrate $(1 \mathrm{~m} \mathrm{~mol}$ each) was considered in the presence of $10 \mathrm{~mol} \%$ of different basic catalysts such as $\mathrm{NaOH}, \mathrm{Ba}(\mathrm{OH})_{2}$, imidazole, and $\mathrm{NaHSO}_{4}$ and ethanol as solvent. We found that, imidazole did not afford the product in good yield and reaction time was very long, similar results were obtained with $\mathrm{Ba}(\mathrm{OH})_{2}$ as basic catalyst. The desired product yield improved to a very less extent when $\mathrm{NaOH}$ was used as a basic catalyst and the product was a sticky mass. When the same reaction was carried out in the presence of $\mathrm{NaHSO}_{4}$, and different solvent such as ethanol, water, water : ethanol in the ratio $1: 1$ and acetonitrile, we found that the desired product improved to a very less extent when ethanol solvent was used but the time still little long then it was not improved when pure water used as solvent, whilst the product yield obtained in very high yield $87 \%$ within 65 mins, when ethanol-water in the ratio $(1: 1)$ used as solvent. In presence of acetonitrile has been used the very poor yield of product has been obtained in long duration time of the reaction because of less polar than water and ethanol and it is aprotic polar solvent. The results of this study are presented in (Table 1). We have also varied the amount of $\mathrm{NaHSO}_{4}$ from $5,7,10$ to $12 \mathrm{~mol} \%$ and the results revealed that, $10 \mathrm{~mol} \%$ gives excellent yield of the product in a short duration as shown in Table 2. When the optimization of the conditions have been done, the generality of this method was examined by the reaction of different substituted aromatic aldehydes with ethyl acetoacetate, 4-fluoro phenylacetonitrile and hydrazine hydrate in the presence of $10 \mathrm{~mol} \% \mathrm{NaHSO}_{4}$ in ethanol-water $(1: 1)$ solvent under reflux; and the results of this study are shown in the Table 3 .

\section{Spectral data analysis}

The structural elucidation of the desired new compounds have been explained using spectronic techniques such as Infra -Red spectrum FT-IR, which give concise view about the functional groups, isomers, geometry, and substituent present in the molecule like $\mathrm{OH}$ group, $\mathrm{NH}_{2}, \mathrm{C} \equiv \mathrm{N}, \mathrm{C} \equiv \mathrm{C}, \mathrm{C}=\mathrm{C}, \mathrm{NO}_{2}, \mathrm{C}=\mathrm{O}, \mathrm{C}-\mathrm{X}$, ortho, meta, para isomers, cis, trans, $\mathrm{CH}_{2}, \mathrm{CH}_{3}$ etc. ${ }^{1} \mathrm{HNMR}$ (Power $400 \mathrm{MHz}$, Solvent DMSO- $d_{6}$ ) spectrum which identified the number of protons exist in the molecule $\mathrm{CH}, \mathrm{CH}_{2}, \mathrm{CH}_{3}$, aromatic protons, aldehyde, carboxylic proton, cis , trans geometry and equivalency in the molecule. ${ }^{13} \mathrm{CNMR}(100 \mathrm{MHz}$, DMSO- $d_{6}$ ) spectrum that used to identify the number of carbon atoms either equivalent or non-equivalent carbon atoms exist in the molecule such as $\mathrm{CH}, \mathrm{CH}_{2}$, $\mathrm{CH}_{3}, \mathrm{C} \equiv \mathrm{N}, \mathrm{C} \equiv \mathrm{C}, \mathrm{C}=\mathrm{C}$ aliphatic or aromatic, $\mathrm{C}=\mathrm{O}$ ester, amide, carboxylic acid, aldehyde, ketone respectively etc. Bellow the spectral data of the synthesised new compounds as they are arranged in Table 3: Yellow crystalline solid (85 \%, $0.590 \mathrm{~g})$; $\mathrm{mp}$ $185-187^{\circ} \mathrm{C}$ : IR (KBr) $v \mathrm{~cm}^{-1}: 3387$ (br), 3065 (ws) 2968 (s), 1694 (vs), 1622 (s), 1375 (s), 1236 (s), 1173 (vs), 1033 (s); Figure 2a. ${ }^{1} \mathrm{HNMR}\left(400 \mathrm{MHz}, \mathrm{DMSO}-d_{6}\right): \delta$ $2.48\left(2 \mathrm{H}, \mathrm{NH}_{2}\right), 3.29(\mathrm{~s}, 3 \mathrm{H}, \mathrm{Me}), 4.89(\mathrm{~s}, 1 \mathrm{H}, \mathrm{CH})$, $6.85-6.38(\mathrm{~d}, \mathrm{~d}, J=8.4 \mathrm{~Hz}, 4 \mathrm{H}, \mathrm{Ph}), 7.84-8.87(\mathrm{~m}$, 
4H, Ph), 10.74 (s, 1H, NH ); Figure 2b. ${ }^{13} \mathrm{CNMR}(100$ $\mathrm{MHz}$, DMSO- $\left.d_{6}\right): \delta 160.0(\mathrm{O}-\mathrm{C}=\mathrm{N}), 157.0,153.0$, 148.1, 135.0, 134.4, 130.0, 122, 115.1 (all ArC), 144.0
( $\mathrm{C}-\mathrm{C}=\mathrm{N}$ pyrazole $), \quad 133.5(\mathrm{O}-\mathrm{C}=\mathrm{C}$ pyrazole $), \quad 115.1$ $(\mathrm{C}=\mathrm{C}$ pyrazole $), 105.3$. $(\mathrm{C}=\mathrm{C}$ pyrano $), 25.1(\mathrm{CH})$, 11.1 $\left(\mathrm{CH}_{3}\right)$. Figure 2c.

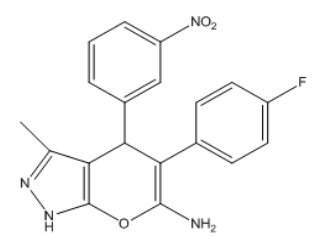

Figure 2: 5- (4-flourophenyl)-3-methyl-4-(3-nitrophenyl)-1,4-dihydropyrano[2,3-c]pyrazol-6-amine (5a).

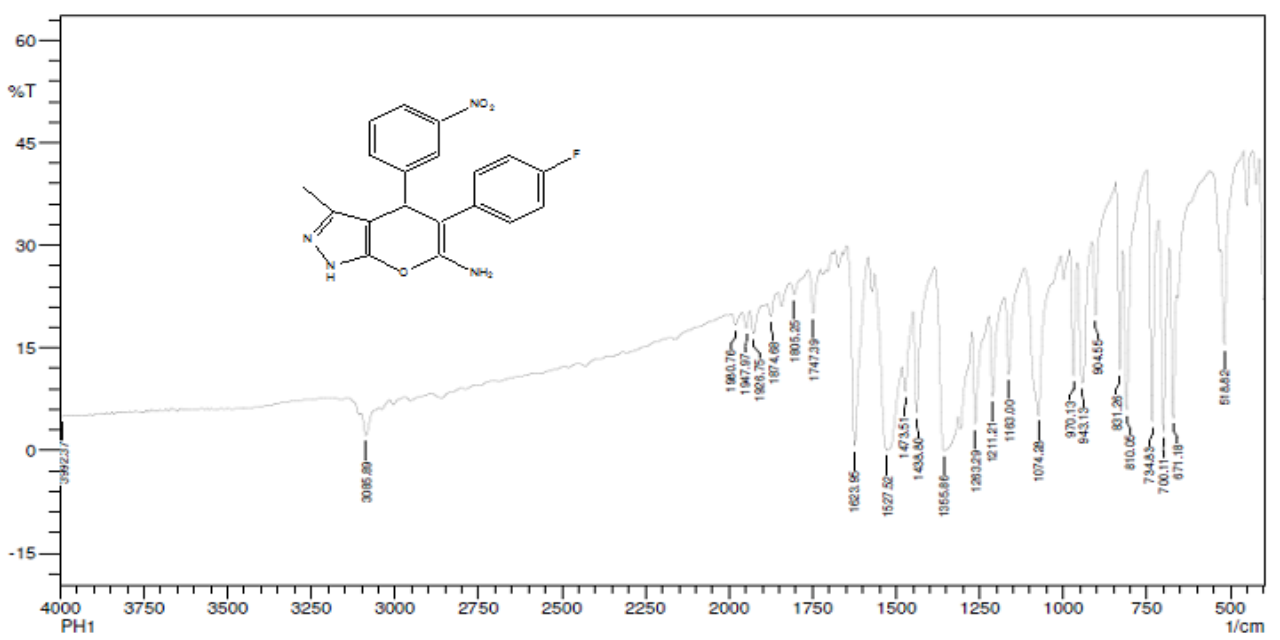

Figure 2a: IR- spectrum.

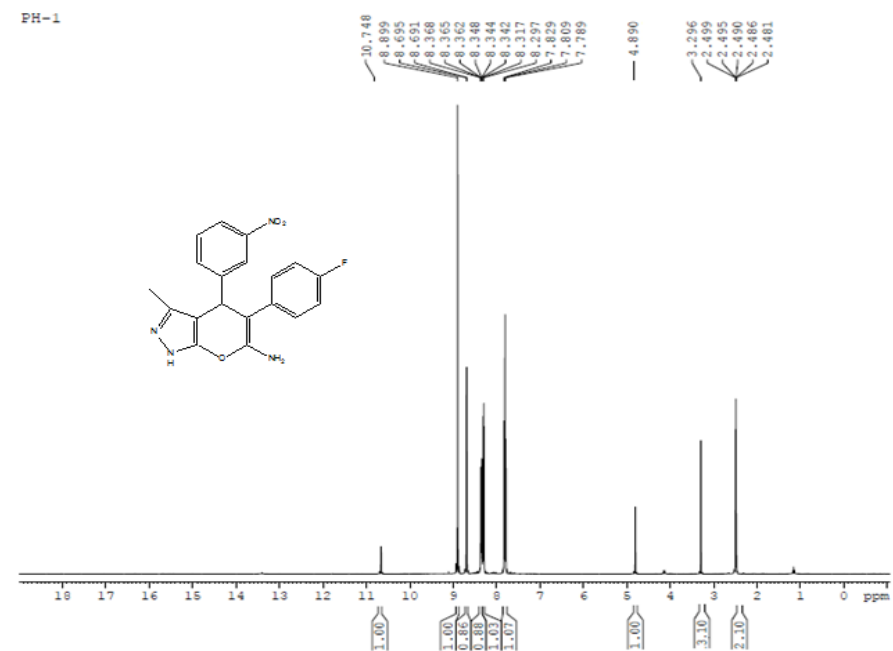

Figure 2b: ${ }^{13} \mathrm{CNMR}$ - spectrum.

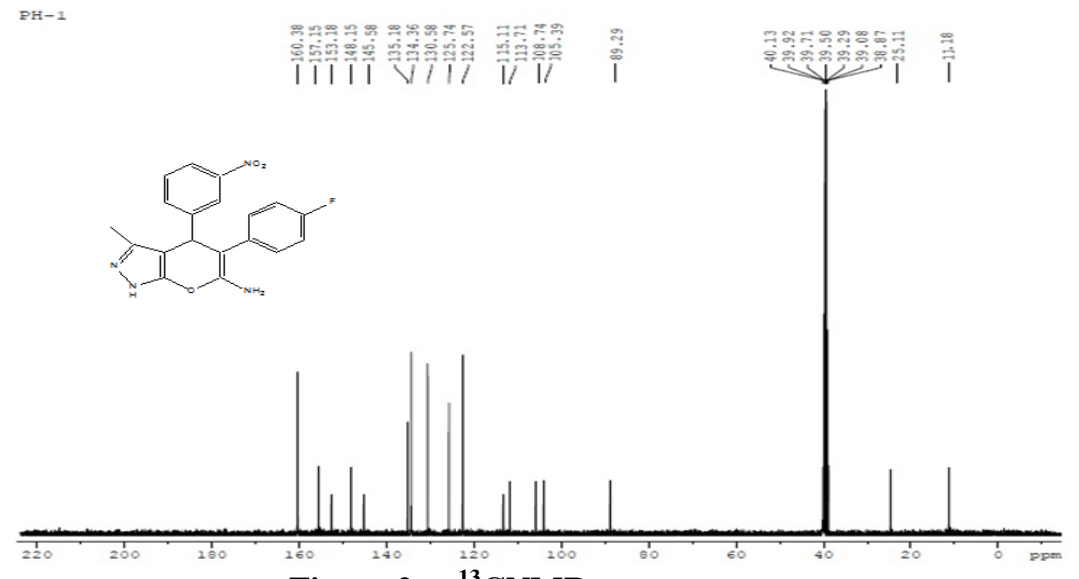

Figure 2c: ${ }^{13}$ CNMR- spectrum. 


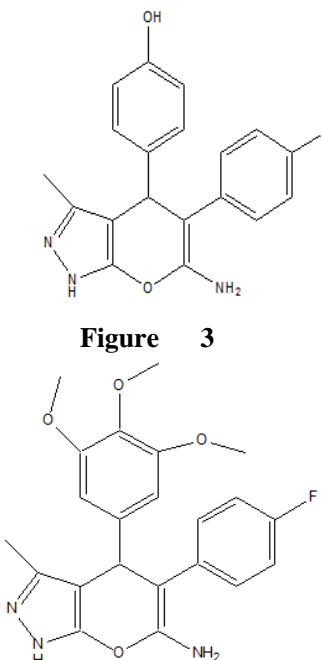

Figure 7<smiles>Cc1n[nH]c2c1C(c1ccc(Cl)cc1)C(c1ccc(F)cc1)=C(N)O2</smiles>

Figure 4<smiles>Cc1n[nH]c2c1C(c1ccc([N+](=O)[O-])cc1)C(c1ccc(F)cc1)=C(N)O2</smiles>

Figure 8<smiles>COc1cc(C2C(c3ccc(F)cc3)=C(N)Oc3[nH]nc(C)c32)ccc1O</smiles>

Figure 5<smiles>Cc1n[nH]c2c1C(c1ccc(Cl)cc1Cl)C(c1ccc(I)cc1)=C(N)O2</smiles>

Figure 9

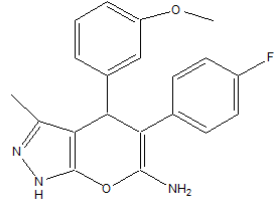

Figure 6

Figure 10<smiles>Cc1n[nH]c2c1C(c1ccccc1O)C(c1ccc(F)cc1)=C(N)O2</smiles>

Figure 11

IUPAC Name

4-(6-amino-5-(4-flourophenyl)-3-methyl-1,4-dihydropyrano[2,3-c]pyrazol-4-yl)phenol (5b) 4-(4-chlorophenyl)-5-(4-flourophenyl)-3-methyl-1,4-dihydropyrano[2,3-c]pyrazol-6-amine (5c) 4-(6-amino-5-(4-flourophenyl)-3-methyl-1,4-dihydropyrano[2,3-c]pyrazol-4-yl)-2-methoxyphenol (5d) 5-(4-flourophenyl)-4-(3-methyoxyphenol)-3-methyl-1,4-dihydropyrano[2,3-c]pyrazol-6-amine (5e) 5-(4-flourophenyl)-4-(3,4,5-trimethyoxyphenol)-3-methyl-1,4-dihydropyrano[2,3-c]pyrazol-6-amine (5f) 5-(4-flourophenyl)-3-methyl-4-(4-nitrophenyl)-1,4-dihydropyrano[2,3-c]pyrazol-6-amine (5g) 4-(2,4-dichlorophenyl)-5-(4-flourophenyl)-3-methyl-1,4-dihydropyrano[2,3-c]pyrazol-6-amine $(5 \mathrm{~h})$ 5-(4-flourophenyl)-4-(3,4-dimethyoxyphenol)-3-methyl-1,4-dihydropyrano[2,3-c]pyrazol-6-amine (5i) 2-(6-amino-5-(4-flourophenyl)-3-methyl-1,4-dihydropyrano[2,3-c]pyrazol-4-yl)phenol (5j)

White crystalline solid (82\%, $0.570 \mathrm{~g}) ; \mathrm{mp} 236-239$ ${ }^{\circ} \mathrm{C}$ : IR (KBr) v: 3534 (br), 3343 (br), 3065 (ws) 2968 (s), 1684 (s), 1598 (s), 1375 (s), 1236 (s), 1173 (vs), 1033 (s), 834 (s) cm ${ }^{-1}$; ${ }^{1} \mathrm{HNMR}$ (400 MHz, DMSO-d 6 ):

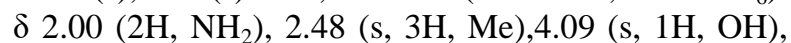
$5.0(\mathrm{~s}, 1 \mathrm{H}, \mathrm{CH}), 6.85-6.38(\mathrm{~d}, \mathrm{~d}, \mathrm{~J}=8.4 \mathrm{~Hz}, 2 \mathrm{H}, \mathrm{Ph})$, $6.85-6.38$ (d,d, J = 8.4 Hz, 4H, Ph ), 10.04 (s, 1H, NH ). White amorphous solid (80 \%, $0.560 \mathrm{~g})$; mp 199-200 ${ }^{\circ} \mathrm{C}$ : IR (KBr) v: 3378 (br), 3047 (ws) 2968 (s), 1684 (s), 1623 (s), 1400 (s), 1235(s), 1163 (vs), 1053 (s), 821 (vs), 653(s ) $\mathrm{cm}^{-1} ;{ }^{1} \mathrm{HNMR}$ (400 MHz, DMSO-d $\left.\mathrm{d}_{6}\right): \delta$ $2.00\left(2 \mathrm{H}, \mathrm{NH}_{2}\right), 2.48$ (s, 3H, Me), $5.0(\mathrm{~s}, 1 \mathrm{H}, \mathrm{CH}), 6.85$ $-6.38(\mathrm{~d}, \mathrm{~d}, \mathrm{~J}=8.4 \mathrm{~Hz}, 2 \mathrm{H}, \mathrm{Ph}), 6.85-6.38(\mathrm{~d}, \mathrm{~d}, \mathrm{~J}=$ $8.4 \mathrm{~Hz}, 4 \mathrm{H}, \mathrm{Ph}), 10.04$ (s, 1H, NH ). ${ }^{13} \mathrm{CNMR}(100$ $\left.\mathrm{MHz}, \mathrm{DMSO}-\mathrm{d}_{6}\right): \delta 160.5(\mathrm{O}-\mathrm{C}=\mathrm{N}), 154.5,151.5$, 143.9, 132.5, 129.0, 120.9, 116.9, 115.6 (all ArC), 143.0 (C-C=N pyrazole), $135.9(\mathrm{O}-\mathrm{C}=\mathrm{C}$ pyrazole $)$,

108.7 ( $\mathrm{C}=\mathrm{C}$ pyrazole $), 104.7(\mathrm{C}=\mathrm{C}$ pyrano $), 23.1$ $(\mathrm{CH}), 11.1\left(\mathrm{CH}_{3}\right)$. Pale orange crystalline solid (87\%, $0.598 \mathrm{~g}$ ); mp $260-263^{\circ} \mathrm{C}$ : IR (KBr) v: 3534 (br), 3452 (s), 3363 (s), 3225 (b), 3065 (ws) 2968 (s), 1680 (s), 1591 (s), 1455 (s), 1423 (s), 1107 (s), 1055 (s), 802 (s), $622(\mathrm{~s}) \mathrm{cm}^{-1} ; \quad{ }^{1} \mathrm{HNMR}\left(400 \mathrm{MHz}, \mathrm{DMSO}-\mathrm{d}_{6}\right): \delta 2.23$ $\left(2 \mathrm{H}, \mathrm{NH}_{2}\right), 2.49$ (s, 3H, Me),3.82 (s, 3H, $\left.\mathrm{OCH}_{3}\right), 5.10$ (s, 1H, OH), $5.65(\mathrm{~s}, 1 \mathrm{H}, \mathrm{CH}), 6.85-6.87(\mathrm{~d}, \mathrm{~d}, \mathrm{~J}=8.4$ $\mathrm{Hz}, 4 \mathrm{H}, \mathrm{Ph}), 7.23-7.53(\mathrm{~d}, \mathrm{~d}, \mathrm{~J}=8.4 \mathrm{~Hz}, 2 \mathrm{H}, \mathrm{Ph})$, $7.44(\mathrm{~s}, 1 \mathrm{H}, \mathrm{Ph}), 8.57$ (s, 1H, NH ); ${ }^{13} \mathrm{CNMR}(100$
MHz, DMSO-d $\left.\mathrm{d}_{6}\right): \delta 161.5(\mathrm{O}-\mathrm{C}=\mathrm{N}), 158.6,150.8$, 148.8, 146.6, 134.9, 126.3, 124.4, 116.4 (all ArC), $140.0 \quad(\mathrm{C}-\mathrm{C}=\mathrm{N}$ pyrazole), $130.0 \quad(\mathrm{O}-\mathrm{C}=\mathrm{C}$ pyrazole), 110.9 ( $\mathrm{C}=\mathrm{C}$ pyrazole), $90.4(\mathrm{C}=\mathrm{C}$ pyrano), 56.4 $\left(\mathrm{OCH}_{3}\right), 23.6(\mathrm{CH}), 12.1\left(\mathrm{CH}_{3}\right)$. Pale yellow amorphous solid (89\%, $0.614 \mathrm{~g})$; mp 195-197 ${ }^{\circ} \mathrm{C}$ : IR (KBr) v: 3484 (w), 3354 (w), 3222 (w), 3014 (ws) 2935 (s), 1675 (s), 1604 (s), 1454 (s), 1387 (s), 1150 (s), 1043 (s), 764 (s) $\mathrm{cm}^{-1} ; \quad{ }^{1} \mathrm{HNMR}$ (400 MHz, DMSO-d 6 ): $\delta 2.48(2 \mathrm{H}$, $\mathrm{NH}_{2}$ ), 3.40 (s, 3H, Me),3.80 (s, 3H, $\left.\mathrm{OCH}_{3}\right), 4.89$ (s, 1H, CH), $7.07-7.09$ (d,d, J = 8.4 Hz, 4H, Ph), $7.38-$ 7.45 (m, 3H, Ph ), 8.67 (s, 1H, Ph), 10.03 (s, 1H, NH ); ${ }^{13}$ CNMR (100 MHz, DMSO-d $\left.{ }_{6}\right): \delta 161.3$ (O-C=N), $159.4,151.5,145.9,138.8,135.1,129.9,125.1,117.4$ (all ArC), $144.4(\mathrm{C}-\mathrm{C}=\mathrm{N}$ pyrazole), $134.0 \quad(\mathrm{O}-\mathrm{C}=\mathrm{C}$ pyrazole), $112.4(\mathrm{C}=\mathrm{C}$ pyrazole $), 88.8(\mathrm{C}=\mathrm{C}$ pyrano), $55.1\left(\mathrm{OCH}_{3}\right), 23.1(\mathrm{CH}), 11.1\left(\mathrm{CH}_{3}\right)$.

Pale orange crystalline solid $(80 \%, 0.560 \mathrm{~g})$; mp 180$184{ }^{\circ} \mathrm{C}$ : IR (KBr) v: $3484(\mathrm{w}), 3354$ (w), $3222(\mathrm{w})$, 3014 (ws) 2986 (s), 1675 (s), 1622 (s), 1438 (s), 1413 (s), 1342 (s), 1234 (s), 1130 (s), 1053 (s), 993 (s), 765 (s), 619 (s) $\mathrm{cm}^{-1} ;{ }^{1} \mathrm{HNMR}$ (400 MHz, DMSO-d $\left.\mathrm{d}_{6}\right): \delta$ $2.48\left(2 \mathrm{H}, \mathrm{NH}_{2}\right), 3.29$ (s, 3H, Me), $3.72\left(\mathrm{~s}, 3 \mathrm{H}, \mathrm{OCH}_{3}\right)$, $3.83\left(\mathrm{~s}, 6 \mathrm{H}, 2 \mathrm{OCH}_{3}\right), 4.64(\mathrm{~s}, 1 \mathrm{H}, \mathrm{CH}), 7.2(\mathrm{~s}, 1 \mathrm{H}, \mathrm{Ph})$, $8.06-8.08(\mathrm{~d}, \mathrm{~d}, J=8.4 \mathrm{~Hz}, 4 \mathrm{H}, \mathrm{Ph}), 8.64(\mathrm{~s}, 1 \mathrm{H}, \mathrm{Ph})$, $10.09(\mathrm{~s}, 1 \mathrm{H}, \mathrm{NH}) ;{ }^{13} \mathrm{CNMR}\left(100 \mathrm{MHz}, \mathrm{DMSO}-d_{6}\right): \delta$ $161.1(\mathrm{O}-\mathrm{C}=\mathrm{N}), 155.4 ， 153.2 ， 144.5,136.4,132.1$, 
129.2, 122.1, 120.1 (all ArC), 144.5 ( $\mathrm{C}-\mathrm{C}=\mathrm{N}$ pyrazole), 132.1 (O-C=C pyrazole), $105.5(\mathrm{C}=\mathrm{C}$ pyrazole $), 88.9$ $(\mathrm{C}=\mathrm{C}$ pyrano $), \quad 60.1\left(\mathrm{OCH}_{3}\right), \quad 55.1\left(2 \mathrm{OCH}_{3}\right), 23.1$ $(\mathrm{CH}), 11.9\left(\mathrm{CH}_{3}\right)$. Pale orange crystalline solid $(82 \%$, $0.570 \mathrm{~g}) ; \mathrm{mp} 271-273{ }^{\circ} \mathrm{C}$ : : IR (KBr) v: $3484(\mathrm{w})$, 3354 (w), 3222 (w), 3114 (ws) 2925 (s), 1675 (s), 1620 (s), 1596 (s), 1523(vs), 1454 (s), 1346 (vs), 1161 (s), 1014 (s), 844 (s) cm ${ }^{-1}$; ${ }^{1} \mathrm{HNMR}$ (400 MHz, DMSO- $d_{6}$ ): $\delta 2.48\left(2 \mathrm{H}, \mathrm{NH}_{2}\right), 3.29$ (s, 3H, Me), $4.84(\mathrm{~s}, 1 \mathrm{H}, \mathrm{CH})$, $8.14-8.16(\mathrm{~d}, \mathrm{~d}, J=8.4 \mathrm{~Hz}, 4 \mathrm{H}, \mathrm{Ph}), 8.35-8.37$ (d,d, $J=8.4 \mathrm{~Hz}, 4 \mathrm{H}, \mathrm{Ph}), 10.05(\mathrm{~s}, 1 \mathrm{H}, \mathrm{NH})$. White crystalline solid $(80 \%, 0.560 \mathrm{~g}) ; \mathrm{mp} 205-207^{\circ} \mathrm{C}$ : : IR (KBr) v: 3448 (w), $3354(\mathrm{w}), 3222(\mathrm{w}), 3089$ (ws) 2935 (s), 1733 (ws), 1616 (s), 1583 (s), 1319 (s), 1139 (s), 1053 (s), 885 (s), 786 (s) cm ${ }^{-1}$; ${ }^{1} \mathrm{HNMR}$ (400 MHz, DMSO-d $\left.d_{6}\right): \delta 2.48\left(2 \mathrm{H}, \mathrm{NH}_{2}\right), 3.28(\mathrm{~s}, 3 \mathrm{H}, \mathrm{Me}), 4.89$ $(\mathrm{s}, 1 \mathrm{H}, \mathrm{CH}), 7.54-7.56(\mathrm{~d}, \mathrm{~d}, J=8.4 \mathrm{~Hz}, 4 \mathrm{H}, \mathrm{Ph}), 8.14$ -8.16 (d,d, $J=8.4 \mathrm{~Hz}, 2 \mathrm{H}, \mathrm{Ph}), 8.96$ (s, 1H, Ph), 11.08 (s, 1H, NH ); ${ }^{13} \mathrm{CNMR}\left(100 \mathrm{MHz}, \mathrm{DMSO}-d_{6}\right): \delta 162.2$ $(\mathrm{O}-\mathrm{C}=\mathrm{N}), 158.1,143.7,141.3,134.6,133.1,130.4$, 128.1, 127.7 (all ArC), 143.7 (C-C=N pyrazole), 130.1 (O-C=C pyrazole), $114.4(\mathrm{C}=\mathrm{C}$ pyrazole $), 88.9(\mathrm{C}=\mathrm{C}$ pyrano), $23.1(\mathrm{CH}), 11.1\left(\mathrm{CH}_{3}\right)$. Orange crystalline solid $(80 \%, 0.560 \mathrm{~g})$; $\mathrm{mp} 175-178^{\circ} \mathrm{C}$ : IR (KBr) v: 3484 (wbr), 3421 (w), 3222 (w), 3002 (ws) 2942 (s),

\section{CONCLUSION}

To conclude, we have reported a new, simple, rapid, and an effective one-pot multi component protocol for the expedient synthesis of dihydro-pyrano [2,3c]pyrazol-6-amines. The noteworthy usefulness of this protocol includes easily available starting materials, simple procedure, and easier separation of products by filtration. The reaction is facile, simple and environment-friendly.

\section{ACKNOWLEDGEMENTS}

One of the authors Dr. S. H. S. Azam sincerely thanks the Department of Chemistry, Faculty of Science, Sana'a University, Sana'a, Yemen for providing necessary facilities; and Prof. M.A. Pasha acknowledges the support from VGST, Dept of IT, BT and S \& T, Govt. of Karnataka, INDIA.

\section{AUTHOR'S CONTRIBUTION}

The manuscript was carried out, written, and approved in collaboration with all authors.

\section{CONFLICT OF INTEREST}

No conflict of interest associated with this work.

\section{REFERENCES}

1. Zhu J, Bienayme H. In Multicomponent Reactions, Wiley: Weinheim 2005

2. Beck B, Hess S, Dömling A. One-pot synthesis and biological evaluation of aspergillamides and analogues. Bioorg. Med Chem Lett 2000; 10(15):1701-1705. https://doi.org/10.1016/s0960-894x(00)00305-x

3. Ugi I. Recent progress in the chemistry of multicomponent reactions. Pure Appl. Chem 2001; 73(1): 187. https://doi.org/10.1351/pac200173010187

4. Hermecz I, Vasvari-Debreczy L, Matyus P. In Comprehensive Heterocyclic Chemistry; Katritzky AR,
1675 (s), 1623 (s), 1508 (s), 1421 (s), 1344 (s), 1271 (s), $1157(\mathrm{~s}), 1018(\mathrm{~s}), 867(\mathrm{~s}), 754(\mathrm{~s}) \mathrm{cm}^{-1}$;

${ }^{1} \mathrm{HNMR}\left(400 \mathrm{MHz}, \mathrm{DMSO}-d_{6}\right)$ : $\delta 2.48\left(2 \mathrm{H}, \mathrm{NH}_{2}\right)$, $3.42(\mathrm{~s}, 3 \mathrm{H}, \mathrm{Me}), 3.81\left(\mathrm{~s}, 6 \mathrm{H}, 2 \mathrm{OCH}_{3}\right), 4.82(\mathrm{~s}, 1 \mathrm{H}$, $\mathrm{CH}), 7.05-7.07$ (d,d, $J=8.4 \mathrm{~Hz}, 4 \mathrm{H}, \mathrm{Ph}), 7.35-7.37$ (d,d, $J=8.4 \mathrm{~Hz}, 2 \mathrm{H}, \mathrm{Ph}), 8.62$ (s, 1H, Ph), 10.09 (s, $1 \mathrm{H}, \mathrm{NH}) ;{ }^{13} \mathrm{CNMR}\left(100 \mathrm{MHz}, \mathrm{DMSO}-d_{6}\right): \delta 160.6$ $(\mathrm{O}-\mathrm{C}=\mathrm{N}), 157.5,151.5,148.9,143.3,135.3,131.4$, 126.6, 123.3 (all ArC), 140.0 (C-C=N pyrazole), 117.5 (O-C=C pyrazole), $115.4(\mathrm{C}=\mathrm{C}$ pyrazole $), 88.8(\mathrm{C}=\mathrm{C}$ pyrano), $55.5\left(\mathrm{OCH}_{3}\right), 55.3\left(\mathrm{OCH}_{3}\right), 23.1(\mathrm{CH}), 11.1$ $\left(\mathrm{CH}_{3}\right)$. White crystalline solid $(82 \%, 0.570 \mathrm{~g}) ; \mathrm{mp}$ 200-204 ${ }^{\circ} \mathrm{C}$ : IR (KBr) v: 3534 (br), 3343 (br), 3065 (ws) 2968 (s), 1684 (s), 1598 (s), 1375 (s), 1236 (s), 1173 (vs), 1033 (s), 834 (s) $\mathrm{cm}^{-1}$;

${ }^{1} \mathrm{HNMR}\left(400 \mathrm{MHz}, \mathrm{DMSO}-d_{6}\right)$ : $\delta 2.00\left(2 \mathrm{H}, \mathrm{NH}_{2}\right)$, $2.48(\mathrm{~s}, 3 \mathrm{H}, \mathrm{Me}), 4.09(\mathrm{~s}, 1 \mathrm{H}, \mathrm{OH}), 5.0(\mathrm{~s}, 1 \mathrm{H}, \mathrm{CH})$, $6.94-6.96(\mathrm{~d}, \mathrm{~d}, J=8.4 \mathrm{~Hz}, 2 \mathrm{H}, \mathrm{Ph}), 7.37-7.41(\mathrm{t}, \mathrm{d}, J$ $=16 \mathrm{~Hz}, 2 \mathrm{H}, \mathrm{Ph}), 7.67-7.69(\mathrm{~d}, \mathrm{~d}, J=8.4 \mathrm{~Hz}, 2 \mathrm{H}, \mathrm{Ph}$ ), $11.09(\mathrm{~s}, 1 \mathrm{H}, \mathrm{NH}) .{ }^{13} \mathrm{CNMR}\left(100 \mathrm{MHz}, \mathrm{DMSO}-d_{6}\right)$ : $\delta 163.2(\mathrm{O}-\mathrm{C}=\mathrm{N}), 159.1,155.5,150.3,149.7,136.7$, 133.7, 131.3, 120.0 (all ArC), 143.3 (C-C=N pyrazole), $118.6(\mathrm{O}-\mathrm{C}=\mathrm{C}$ pyrazole $), \quad 117.0(\mathrm{C}=\mathrm{C}$ pyrazole $), 89.9$ $(\mathrm{C}=\mathrm{C}$ pyrano $), 23.0(\mathrm{CH}), 11.1\left(\mathrm{CH}_{3}\right)$.

Rees CW, Scriven EVF, Eds Pergamon: London, 1996; Chapter 8. 23: 563.

5. Jayaraman M, Fox BM, Hollingshead M, Kohlhagen G, Pommier Y, Cushman M. Synthesis of new dihydroindeno[1,2-c]isoquinoline and indenoisoquinolinium chloride. J Med Chem 2002; 45(1): 242-249. https://doi.org/10.1021/jm000498f

6. Goldberg DR, et al. Optimization of 2- phenylaminoimidazo[4,5-h]isoquinolin-9-ones: orally active inhibitors of lck kinase. J Med Chem 2003; 46(8):1337-49. https://doi.org/10.1021/jm020446l

7. Griffin RJ, et al. Selective benzopyranone and pyrimido[2,1-a]isoquinolin-4-one inhibitors of DNAdependent protein kinase: synthesis, structure-activity studies, and radio sensitization of a human tumor cell line in vitro. J Med Chem 2005; 48(2): 569-585. https://doi.org/10.1021/jm049526a

8. Gold brunner M, Loidl G, Polossek T, Mannschreck, A, von Angerer E. Inhibition of tubulin polymerization by 5,6-dihydroindolo[2,1-alpha]isoquinoline derivatives. J Med Chem 1997; 40(22): 3524-3533. https://doi.org/10.1021/jm970177c

9. Ruppert D, Weithmann KU. HL 725, an extremely potent inhibitor of platelet phosphodiesterase and induced platelet aggregation in vitro. Life Sci 1982; 31(19): 2037-2043. https://doi.org/10.1016/0024-3205(82)90095-9

10. Swinbourne JF, Hunt HJ, Klinkert G. One-Pot threecomponent synthesis of oxazine derivatives in water. Adv Heterocycl Chem 1987; 23, 103-13. http://dx.doi.org/10.5155/eurjchem.5.3.541-544.1049

11. Anastas PT, Warner, J.C. Green Chemistry: Theory and Practice, Oxford University Press, Oxford, 2000.

12. Poliakoff M, Licence P. Modern life depends on the petrochemical industry most drugs, paints and plastics derive from oil. But current processes for making chemical products are not sustainable in terms of resources and environmental impact. Green chemistry aims to tackle this problem, and real progress is being made. Nature 2007; 450(7171): 810-812. https://doi.org/10.1038/450810a

13. Clark JH. Green chemistry: today (and tomorrow). Green Chem 2006; 8: 17-21. https://doi.org/10.1039/B516637N 
14. Li CJ, Chen L. Organic chemistry in water. Chem Soc Rev 2006; 35: 68-82. https://doi.org/10.1039/B507207G

15. Polshettiwar V, Varma RS. Olefin ring closing metathesis and hydrosilylation reaction in aqueous medium by Grubbs second generation ruthenium catalyst. J Org Chem 2008; 73(18): 7417-7419. https://doi.org/10.1021/jo801330c

16. Polshettiwar V, Varma RS. Tandem Bis-aldol reaction of ketones: a facile one-pot synthesis of 1,3-Dioxanes in Aqueous Medium. J Org Chem 2007; 72(19): 74207422. https://doi.org/10.1021/jo701337j

17. Polshettiwar V, Varma RS. Microwave-Assisted Organic Synthesis and Transformations using Benign Reaction. Chem Soc Rev 2008; 37: 1546-1557. https://doi.org/10.1039/B716534J

18. Dallinger D, Kappe, CO. Microwave- assisted synthesis in water as solvent. Chem Rev 2007; 107: 2563-2591. https://doi.org/10.1021/cr0509410

19. Pol-shettiwar V, Varma RS. Alternative Heating for Green Synthesis in Water (Photo, Ultrasound, and Microwave), Hand book of Green Chemistry, ed. Anastas, P. T.; Li, C.-J. Wil VCH Verlag GmbH, Weinheim, 2009.

20. Varma RS. Clean chemical synthesis in water, Org Chem. Highlights 2007.

21. Gnanasambandam V, Kandhasamy K. Four-component catalyst-free reaction in water: Combinatorial library synthesis of novel 2-amino-4-(5-hydroxy-3-methyl-1Hpyrazol-4-yl)-4H-chromene-3-carbonitrile derivatives. Green Chem 2009; 11(12): 1945-1947. https://doi.org/10.1039/B913838B

22. Bigi F, Chesini L, Maggi R, Sartori G. Montmorillonite $\mathrm{KSF}$ as an Inorganic, Water Stable, and Reusable Catalyst for the Knoevenagel Synthesis of Coumarin-3-carboxylic Acids. J Org Chem 1999; 64(3): 10331035. https://doi.org/10.1021/jo981794r

23. Bigi F, Conforti ML, Maggi R, Piccinno A, Sartori G. Clean Synthesis in Water: Uncatalysed Preparation of Ylidenemalonitriles. Green Chem 2000; 2:101-103. http://dx.doi.org/10.1039/b001246g

24. Kuppusamy K, KasinP. Solvent-free multicomponent synthesis of pyranopyrazoles: per-6-amino- $\beta$-cyclodextrin as a remarkable catalyst and host. Tetrahedron Lett 2010; 51(25): 3312-3316.

25. El-Tamany ES, El-Shahed FA, Mohamed BH. Synthesis and biological activity of some pyrazole derivatives. J Serb Chem Soc 1999; 64: 9-18.

26. Ismail ZH, Aly GM, El-Degwi MS, Heiba HI, Ghorab MM. Egypt J Biot 2003; 13: 73.

27. Zaki MEA, Soliman HA, Hiekal OA, Rashad AEZ. Pyrazolo pyranopyrimidines as a class of antiinflammatory agents and some of them revealed moderate to potent antiinflammatory activity. Naturforsch 2006; 61: 1-5. https://doi.org/10.1515/znc-2006-1-201

28. Wang JL, Liu D, Zhang ZJ, Shan S, Han X, Srinivasula SM, Croce C M, Alnemri ES, Huang Z. Structure-based discovery of an organic compound that binds Bcl-2 protein and induces apoptosis of tumor cells. Proc Natl Acad Sci USA 2000; 97(13): 7124-7129. https://doi.org/10.1073/pnas.97.13.7124

29. Abdelrazek FM, Metz P, Metwally NH, El-Mahrouky SF. Synthesis and molluscicidal activity of new cinnoline and pyrano[2,3-c]pyrazole derivatives. Arch Pharm Chem Life Sci 2006; 339: 456-460. https://doi.org/10.1002/ardp.200600057

30. Sharanin YA, Sharanina LG, Puzanova VV. Nitrile cyclization reactions. VII. Synthesis of 6-amino-4-aryl-3methyl-5-cyano-1H,4H-pyrazolo[3,4-b]pyrans. Zh Org Khim 1983; 19: 2609-2615.
31. Rodinovskaya LA, Gromova AV, Shestopalov AM, Nesterov VN. Synthesis of 6-amino-4-aryl-5-cyano-3-(3cyanopyridin-2-ylthiomethyl)-2,4-dihydropyrano [2,3-c] pyrazoles and their hydrogenated analogs. Molecular structure of 6-amino-5-cyano- (3-cyano-4,6-dimethylpyridin-2-ylthiomethyl)-4-(2-nitrophenyl)-2,4dihydropyrano[2,3-c]pyrazole. Russ Chem Bull Int Ed 2003; 52: 2207-2213. https://doi.org/10.1023/B:RUCB.0000011880.05561.c1

32. Junek H, Aigner H. Synthesen mit Nitrilen, XXXV. Reaktionen von Tetracyanäthylen mit Heterocyclen. Chem Ber 1973; 106(3): 914-921. https://doi.org/10.1002/cber.19731060323

33. Abdelrazek FM, Metz P, Kataeva O, Jaeger A, ElMahrouky SF. Synthesis and molluscicidal activity of new chromene and pyrano[2,3-c]pyrazole derivatives. Arch Pharm 2007; 340(10): 543-548. DOI: 10.1002/ardp.200700157. (b) Foloppe N, Fisher LM, Howes R, Potter A, Robertson AGS, Surgenor AE. Identification of chemically diverse Chk1 inhibitors by receptor-based virtual screening. Bioorg Med Chem 2006; 14(14): 4792-4802. https://doi.org/10.1016/j.bmc.2006.03.021

34. Fadda A A, Abdel-Rahman AAH, Hamed EA, Khalil EH. Utility of Enaminonitriles in Heterocyclic Synthesis: Synthesis and Antimicrobial Activity of Some New Azole and Azine Derivatives. Am Jr Org Che 2012; 2: 7-13. https://doi.org/10.5923/j.ajoc.20120202.02

35. Jaberi ZK, Shams M M R, Pooladian B. Expeditious, Four-Component Synthesis of 1,4-Dihydropyrano[2,3c]Pyrazole Derivatives Catalyzed by Trichloroacetic Acid or Ceric Sulfate. Acta Chimica Slovenica 2013; 60(1):105108

36. Kanchithalaivan S, Sivakumar S, Kumar RR. Elumalai, P.; Ahmed, Q. N.; Padala A. K. Four-component domino strategy for the combinatorial synthesis of novel 1, 4dihydropyrano[2,3-c]pyrazol-6-amines. ACS Comb Sci 2013; 15(12): 631-638

37. Madhusudana Reddy MB, Pasha MA. Molecular iodinecatalyzed, mild, effective, ecofriendly, microwaveassisted, one-pot synthesis of 5-arylmethylidene-2phenyloxazol-4-ones (azalactones) under solvent-free. Synth Commun 2010; 40: 1895-1898. https://doi.org/10.1080/00397910903162783

38. Pasha M A, Jayashankara VP. Molecular iodine catalyzed synthesis of aryl-14H-dibenzo[a,j]xanthenes under solvent free condition. Bioorg Med Chem Lett 2007; 17(3): 621-623. https://doi.org/10.1016/j.bmcl.2006.11.009

39. Pasha MA, Jayashankara VP. An Efficient Synthesis of 2Aminobenzochromene Derivatives Catalyzed by Tetrabutyl ammoniumbromide (TBABr) under Microwave Irradiation in Aqueous Medium. Indian J Chem 2007; 46B: $1328-1331$ http://dx.doi.org/10.1002/chin.200750152

40. Madhusudana Reddy. M. B.; Pasha, M. A. Environment friendly protocol for the synthesis of nitriles from aldehydes. Chin Chem Lett 2010; 21(9): 1025. https://doi.org/10.1016/j.cclet.2010.05.004

41. Pasha MA, Jayashankara VP. An expeditious synthesis of 15 benzodiazepine derivatives catalyzed by $p$ toluene sulfonic acid. J Pharm Toxic 2006; 1(6), 573-578.

42. Pasha MA, Aatika N. Iodine-Catalyzed, Rapid and Efficient, One-Pot Synthesis of 1, 2-Dihydro-1arylnaphtho[1,2e][1,3]oxazine-3-ones Under Solvent-Free Conditions. Synth Commun 2010; 40(19): 2864-2868. https://doi.org/10.1080/00397910903320654

43. Aatika N, Pasha MA. p-TSA catalysed efficient synthesis of 2, 4, 5-tetraaryl-imidazoles. J Saudi Chem Soc 2011; 15: 55-58. https://doi.org/10.1016/j.jscs.2011.01.004 